\title{
Two New Cytotoxic Candidaspongiolides from an Indonesian Sponge
}

\author{
Agus Trianto, ${ }^{1,2}$ Idam Hermawan, ${ }^{1}$ Toshimasa Suzuka, ${ }^{1}$ and Junichi Tanaka ${ }^{1}$ \\ ${ }^{1}$ Department of Chemistry, Biology and Marine Science, University of the Ryukyus, Senbaru 1, Nishihara, Okinawa 903-0213, Japan \\ ${ }^{2}$ Department of Marine Sciences, Faculty of Fisheries and Marine Sciences, \\ University of Diponegoro, Semarang, Central Java 50234, Indonesia
}

Correspondence should be addressed to Agus Trianto, trianto_telawur@yahoo.co.id

Received 2 May 2011; Accepted 27 May 2011

Academic Editors: T. Schotten and X.-W. Yang

Copyright (c) 2011 Agus Trianto et al. This is an open access article distributed under the Creative Commons Attribution License, which permits unrestricted use, distribution, and reproduction in any medium, provided the original work is properly cited.

\begin{abstract}
Marine sponges have been recognized as potentially rich sources of various bioactive molecules. In our continuing search for new secondary metabolites from Indonesian marine invertebrates, we collected a sponge, whose extract showed cytotoxicity against cultured cells at $0.1 \mu \mathrm{g} / \mathrm{mL}$. Purification of the extract yielded two new macrolides 2 and 3 along with known candidaspongiolide (1). The structures for compounds 2 and 3 were elucidated by spectral analysis $\left({ }^{1} \mathrm{H},{ }^{13} \mathrm{C}\right.$, COSY, HMQC, HMBC) and by comparison of their NMR data with those of $\mathbf{1}$. Compounds 2 and 3 exhibited a little more potent cytotoxicity $\left(\mathrm{IC}_{50} 4.7\right.$ and $19 \mathrm{ng} / \mathrm{mL}$ ) than that $\left(\mathrm{IC}_{50} 37 \mathrm{ng} / \mathrm{mL}\right)$ of candidaspongiolide (1) against NBT-T2 cells.
\end{abstract}

\section{Introduction}

Sponges, a group of sedentary organisms, cannot move and escape from predators. Most sponges are filter feeders pumping water to its body to obtain foods and oxygen and to expel wastes and may be threatened by microorganisms during filtering seawater rich in bacteria and fungi $[1,2]$. In order to defend themselves against predators, pathogens and competitors, sponges may have developed to produce or accumulate secondary metabolites during their long evolution, such as feeding deterrent, antimicrobial, antifungal, and antifouling molecules. Interestingly, some of the compounds have also shown remarkable potency as drug candidates against various human diseases as discussed elsewhere [3-9].

In 1984, Schmitz and coworkers isolated tedanolide from the Caribbean marine sponge Tedania ignis [10]. Tedanolide is a unique 18-membered macrolide where lactonization occurs at a primary hydroxyl group instead of a common secondary one, and this class of macrolide has been reported to exhibit strong cytotoxicity at pico to nanomolar range [10, 11]. The unique structure in combination with promising biological activity leads tedanolide as an intriguing target for formal and total syntheses [12-14]. More recently, Meragelman and coworkers reported a macrolide named candidaspongiolide (1) related to tedanolide with modification at C-11 to C-15 from the marine sponge Candidaspongia sp. Candidaspongiolide exhibited potent cytotoxicity in NCI 60 cells panel with $\mathrm{GI}_{50}$ of $14 \mathrm{ng} / \mathrm{mL}$ [15], protein synthesis inhibition, and apoptosis induction [16].

In our continuing search for potential drug leads from Indonesian marine invertebrates $[17,18]$, we obtained a sponge whose extract showed cytotoxicity at $0.1 \mu \mathrm{g} / \mathrm{mL}$ against NBT-T2 cells in a screening process. Purification of the extract provided candidaspongiolide (1) along with two new analogs $\mathbf{2}$ and $\mathbf{3}$, which are the subject of this paper.

\section{Materials and Methods}

2.1. Chemicals and Equipments. Methanol $(\mathrm{MeOH})$ used for extraction was of technical grade. Reagent grade solvents were used for isolating compounds $\mathbf{1 - 3}$. Merck Si-60 (70230 mesh) was used for silica gel column chromatography, while Merck Si-60 $\mathrm{F}_{254}$ for analytical TLC. HPLC was performed either on a Waters 510 pump with a Waters 486 UV detector and a Shodex RI-101 or on a Hitachi L-6000 pump with a Hitachi L-4000 UV detector and a Shodex RI-101 using a Mightysil Si-60 $(10 \times 250 \mathrm{~mm})$ 
column. Optical rotations were measured on a Jasco P1010 polarimeter using a cell with $3.5 \mathrm{~mm}$ aperture. IR spectra were recorded on a Jasco FT/IR-6100 instrument, whereas HRESIMS was measured on a Jeol JMS-T100LP spectrometer using reserpine or sodium trifluoroacetate as an internal standard. Most of ${ }^{1} \mathrm{H}$ and ${ }^{13} \mathrm{C}$ NMR spectra were measured in $\mathrm{CDCl}_{3}$, while those of compound 3 were measured in $\mathrm{CD}_{3} \mathrm{OD}$ with TMS as an internal standard on a Jeol A500 and/or a Bruker AVANCE III-500 in $\mathrm{CDCl}_{3}$. The ${ }^{1} \mathrm{H}$ and ${ }^{13} \mathrm{C}$ chemical shifts were given in ppm, while coupling constants were in $\mathrm{Hz}$.

2.2. Sponge. Specimens of the sponge tagged K09-02 was collected by hand using SCUBA at 15-25 m depth at Kupang, West Timor, East Nusa Tenggara, Indonesia on August 2009. By comparing underwater images of our specimen with that of the specimen of NCI group [15], it is likely to be the same sponge. The specimen was kept frozen until extraction. The sponge K09-02 may be an endemic species to this region. The colonies are grey in color and stand.

2.3. Extraction and Isolation. After cutting into small pieces, the sponge ( $653 \mathrm{~g}$, wet) was soaked in $\mathrm{MeOH}$ for $24 \mathrm{~h}$ for three times. Then, the solution was concentrated under vacuum to obtain a crude extract. The methanolic extract (17.0 g) was triturated with ethyl acetate (EtOAc) to provide a lipophilic fraction $(2.7 \mathrm{~g})$, which killed NBT-T2 cells at $0.1 \mu \mathrm{g} / \mathrm{mL}$. This fraction was subjected to a silica gel column eluting with stepwise gradient solvents (hexane: EtOAc = $2: 1,1: 1,1: 2,0: 1$, EtOAc: $\mathrm{MeOH}=10: 1)$ to afford ten fractions. Fraction $5(126.0 \mathrm{mg})$ was purified by repetitive Si-60 HPLC using hexane-EtOAc mixtures to provide candidaspongiolide 1 (15.4 mg). Fraction 6 (70.8 mg) was also purified by Si-60 HPLC using a solvent system hexane $:$ EtOAc $=2: 1$ to afford compound $2(9.8 \mathrm{mg})$. Fraction 9 (107.1 mg) was also subjected to repetitive Si-60 HPLC using hexane : EtOAc = 1:6, EtOAc: $\mathrm{CH}_{2} \mathrm{Cl}_{2}: \mathrm{MeOH}=20: 20: 1$, and EtOAc: $\mathrm{CH}_{2} \mathrm{Cl}_{2}: \mathrm{MeOH}=10: 20: 1$ as solvent systems sequentially, to give compound $3(21.8 \mathrm{mg})$. Isolation of these compounds was guided by cytotoxicity testing and NMR spectra.

2.4. Compound 1. Colorless glass, $[\alpha]_{\mathrm{D}}^{25}+69(c 0.55, \mathrm{MeOH})$. IR $v_{\max }$ (neat) 3419, 2925, 2854, 1742, 1715, 1456, 1372, 1234, $1086 \mathrm{~cm}^{-1} .{ }^{1} \mathrm{H}$ and ${ }^{13} \mathrm{C}$ NMR; see Tables 1 and 2. HR-ESIMS $[\mathrm{M}+\mathrm{Na}]^{+} \mathrm{m} / z$ 945.55514, 959.57079, 973.58884 (calcd for $\mathrm{C}_{50} \mathrm{H}_{82} \mathrm{NaO}_{15}{ }^{+} 945.55459(\Delta+0.58 \mathrm{ppm})$, $\mathrm{C}_{51} \mathrm{H}_{84} \mathrm{NaO}_{15}{ }^{+} 959.57024$ (+0.57 ppm), and $\mathrm{C}_{52} \mathrm{H}_{86} \mathrm{NaO}_{15}{ }^{+}$ $973.58589(+3.0 \mathrm{ppm}))$.

2.5. Compound 2. Colorless glass, $[\alpha]_{\mathrm{D}}^{25}+72(c 0.75, \mathrm{MeOH})$. IR $v_{\max }$ (neat) $3421,2925,2854,1748,1715,1456 \mathrm{~cm}^{-1} \cdot{ }^{1} \mathrm{H}$ and ${ }^{13} \mathrm{C}$ NMR; see Tables 1 and 2. HR-ESIMS $[\mathrm{M}+\mathrm{Na}]^{+} \mathrm{m} / z$ 903.54964, 917.56023, 931.57576, 945.60036 and 959.61056 (calcd for $\mathrm{C}_{48} \mathrm{H}_{80} \mathrm{NaO}_{14}{ }^{+} 903.54403(\Delta+6.2 \mathrm{ppm})$, $\mathrm{C}_{49} \mathrm{H}_{82} \mathrm{NaO}_{14}{ }^{+} 917.55968$ (+0.60 ppm), $\mathrm{C}_{50} \mathrm{H}_{84} \mathrm{NaO}_{14}{ }^{+}$ 931.57533 (+0.46 ppm), $\mathrm{C}_{51} \mathrm{H}_{86} \mathrm{NaO}_{14}{ }^{+} 945.59098$ (+9.9 ppm), and $\left.\mathrm{C}_{52} \mathrm{H}_{88} \mathrm{NaO}_{14}{ }^{+} 959.60663(+4.1 \mathrm{ppm})\right)$.
2.6. Compound 3. Yellow glass, $[\alpha]_{\mathrm{D}}^{25}+97(c 0.35, \mathrm{MeOH})$. IR $\nu_{\max }$ (neat) $3418,2925,2854,1715,1457,1373,1244$, 1084, $995 \mathrm{~cm}^{-1} \cdot{ }^{1} \mathrm{H}$ and ${ }^{13} \mathrm{C}$ NMR; see Tables 1 and 2 . HRESIMS $[\mathrm{M}+\mathrm{Na}]^{+} \mathrm{m} / z 665.31522$ (calcd for $\mathrm{C}_{32} \mathrm{H}_{50} \mathrm{NaO}_{13}{ }^{+}$ $665.31436(+1.3 \mathrm{ppm}))$.

2.7. Acetylation. Compound $\mathbf{1}(0.2 \mathrm{mg})$ was dissolved in pyridine $(50 \mu \mathrm{L})$ and acetic anhydride $(50 \mu \mathrm{L})$. The mixture was stirred for three days under a nitrogen atmosphere at room temperature. After removal of excess reagents with nitrogen flow and vacuum, the reaction product 4 was checked with ${ }^{1} \mathrm{H}$ NMR and ESIMS. Compound 2 was similarly treated to give 4 .

2.8. Compound 4 from 1. ${ }^{1} \mathrm{H}$ NMR: $\delta 5.65 \mathrm{dd}(J=2.7$, $9.5 \mathrm{~Hz}), 5.52 \mathrm{~m}, 5.51 \mathrm{~d}(J=10.1 \mathrm{~Hz}), 5.36 \mathrm{~m}, 5.34 \mathrm{dd}(J=$ $2.2,9.1 \mathrm{~Hz}), 5.28 \mathrm{dt}(J=2.3,10.5 \mathrm{~Hz}), 5.07 \mathrm{~d}(J=11.0 \mathrm{~Hz})$, $4.79 \mathrm{~d}(J=6.6 \mathrm{~Hz}), 4.54 \mathrm{dd}(J=2.0,11.3 \mathrm{~Hz}), 4.25 \mathrm{dd}$ $(J=2.3,8.1 \mathrm{~Hz}), 4.13 \mathrm{dd}(J=6.3,11.5 \mathrm{~Hz}), 3.43 \mathrm{~s}(3 \mathrm{H})$, $3.34 \mathrm{dq}(J=10.0,6.7 \mathrm{~Hz}), 3.29 \mathrm{dq}(J=10.5,7.0 \mathrm{~Hz}), 3.01 \mathrm{dd}$ $(J=9.2,18.4 \mathrm{~Hz}), 2.88 \mathrm{~d}(J=9.3 \mathrm{~Hz}), 2.60 \mathrm{dd}(J=2.6$, $18.4 \mathrm{~Hz}), 2.35-2.4 \mathrm{~m}, 2.32 \mathrm{dd}(J=1.9,9.3 \mathrm{~Hz}), 2.21 \mathrm{~s}(3 \mathrm{H})$, $2.10 \mathrm{~s}(3 \mathrm{H}), 2.09 \mathrm{~s}(3 \mathrm{H}), 2.02 \mathrm{~s}(3 \mathrm{H}), 1.69 \mathrm{~d}(J=1.1 \mathrm{~Hz}$, $3 \mathrm{H}), 1.66 \mathrm{dd}(J=1.7,6.8 \mathrm{~Hz}, 3 \mathrm{H}), 1.44 \mathrm{~d}(J=7.1 \mathrm{~Hz}, 3 \mathrm{H})$, $1.37 \mathrm{~s}(3 \mathrm{H}), 1.17 \mathrm{~d}(J=7.1 \mathrm{~Hz}, 3 \mathrm{H}), 1.08 \mathrm{~d}(J=6.6 \mathrm{~Hz}$, $3 \mathrm{H}), 0.90 \mathrm{t}(\mathrm{J}=6.1 \mathrm{~Hz}, 3 \mathrm{H})$. HR-ESIMS $m / z$ 1072.60225, 1086.60997, 1099.62465 (calcd for ${ }^{12} \mathrm{C}_{55}{ }^{13} \mathrm{CH}_{88} \mathrm{NaO}_{18}{ }^{+}$ 1072.59019 (+11.24 ppm), ${ }^{12} \mathrm{C}_{56}{ }^{13} \mathrm{CH}_{90} \mathrm{NaO}_{18}{ }^{+} 1086.60584$ (+3.8 ppm), and $\mathrm{C}_{58} \mathrm{H}_{92} \mathrm{NaO}_{18}{ }^{+} 1099.61814$ (+5.9 ppm)).

2.9. Compound 4 from 2. ${ }^{1} \mathrm{H}$ NMR: $\delta 5.65 \mathrm{dd}(J=2.6$, $9.8 \mathrm{~Hz}), 5.52 \mathrm{~m}, 5.51 \mathrm{~d}(J=10.2 \mathrm{~Hz}), 5.37 \mathrm{~m}, 5.34 \mathrm{dd}(J=$ $1.7,9.1 \mathrm{~Hz}), 5.28 \mathrm{dt}(J=1.7,10.5 \mathrm{~Hz}), 5.07 \mathrm{~d}(J=10.9 \mathrm{~Hz})$, $4.78 \mathrm{~d}(J=6.3 \mathrm{~Hz}), 4.54 \mathrm{dd}(J=3.1,11.7 \mathrm{~Hz}), 4.25 \mathrm{dd}(J=$ $2.3,8.1 \mathrm{~Hz}), 4.13 \mathrm{dd}(J=6.5,11.3 \mathrm{~Hz}), 3.43 \mathrm{~s}(3 \mathrm{H}), 3.34 \mathrm{dq}(J$ $=10.1,5.7 \mathrm{~Hz}), 3.29 \mathrm{dq}(J=10.5,7.2 \mathrm{~Hz}), 3.01 \mathrm{dd}(J=9.4$, $18.6 \mathrm{~Hz}), 2.88 \mathrm{~d}(J=9.2 \mathrm{~Hz}), 2.60 \mathrm{dd}(J=2.5,18.6 \mathrm{~Hz}), 2.35-$ $2.4 \mathrm{~m}, 2.32(J=1.9,9.3 \mathrm{~Hz}), 2.22 \mathrm{~s}(3 \mathrm{H}), 2.10 \mathrm{~s}(3 \mathrm{H}), 2.09 \mathrm{~s}$ $(3 \mathrm{H}), 2.02 \mathrm{~s}(3 \mathrm{H}), 1.70 \mathrm{~d}(J=1.1 \mathrm{~Hz}, 3 \mathrm{H}), 1.62 \mathrm{dd}(J=1.7$, $6.8 \mathrm{~Hz}, 3 \mathrm{H}), 1.44 \mathrm{~d}(J=7.1 \mathrm{~Hz}, 3 \mathrm{H}), 1.37 \mathrm{~s}(3 \mathrm{H}), 1.17 \mathrm{~d}(J$ $=7.1 \mathrm{~Hz}, 3 \mathrm{H}), 1.08 \mathrm{~d}(J=6.6 \mathrm{~Hz}, 3 \mathrm{H}), 0.90 \mathrm{t}(J=6.1 \mathrm{~Hz}$, $3 \mathrm{H})$. HR-ESIMS $\left[\mathrm{M}+\mathrm{Na}^{+}\right] \mathrm{m} / z$ 1071.58258, 1085.60199, 1099.61165 (calcd for $\mathrm{C}_{56} \mathrm{H}_{88} \mathrm{NaO}_{18}{ }^{+} 1071.58629$ (-4 ppm), $\mathrm{C}_{57} \mathrm{H}_{90} \mathrm{NaO}_{18}{ }^{+} 1085.60194(-.5 \mathrm{ppm})$, and $\mathrm{C}_{58} \mathrm{H}_{92} \mathrm{NaO}_{18}{ }^{+}$ $1099.61759(-5.9 \mathrm{ppm}))$.

2.10. Screening Process. NBT-T2 cells were purchased from Riken and used for cytotoxicity testing. NBT-T2 is a cell line derived from chemically induced rat bladder carcinoma cells [19]. The sponge extract was tested at $0.1,1$, and $10 \mu \mathrm{g} / \mathrm{mL}$ in triplicate, while fractions were done at 0.01 , 0.1 , and $1 \mu \mathrm{g} / \mathrm{mL}$. The cells were cultured in Dulbecco's modified Eagle's medium (DMEM) supplemented with Sigma antibiotic-antimycotic, Biowest fetal bovine serum, Gibco MEM nonessential amino acid in a Falcon 24-well plate or 48-well plate. After adding the extract or a fraction, cells were incubated for $24 \mathrm{~h}$ under $5 \% \mathrm{CO}_{2}$ at $36^{\circ} \mathrm{C}$ [16]. 
TABle 1: ${ }^{13} \mathrm{C}$ NMR data for compounds $\mathbf{1}, \mathbf{2}$, and 3.

\begin{tabular}{|c|c|c|c|c|}
\hline C no. & $\mathbf{1}^{15, \mathrm{a}}$ & $1^{\mathrm{a}}$ & $2^{\mathrm{a}}$ & $3^{\mathrm{b}}$ \\
\hline 1 & $171.3 \mathrm{qC}$ & $171.3 \mathrm{qC}$ & $171.3 \mathrm{qC}$ & $171.3 \mathrm{qC}$ \\
\hline 2 & $70.7 \mathrm{CH}$ & $70.8 \mathrm{CH}$ & $70.8 \mathrm{CH}$ & $72.9 \mathrm{CH}$ \\
\hline 3 & $83.4 \mathrm{CH}$ & $83.3 \mathrm{CH}$ & $83.1 \mathrm{CH}$ & $84.7 \mathrm{CH}$ \\
\hline 4 & $47.8 \mathrm{CH}$ & $47.7 \mathrm{CH}$ & $47.9 \mathrm{CH}$ & $49.5 \mathrm{CH}$ \\
\hline 5 & $214.6 \mathrm{qC}$ & $214.9 \mathrm{qC}$ & $216.2 \mathrm{qC}$ & $217.6 \mathrm{qC}$ \\
\hline 6 & $48.5 \mathrm{CH}$ & $48.3 \mathrm{CH}$ & $49.8 \mathrm{CH}$ & $51.1 \mathrm{CH}$ \\
\hline 7 & $80.1 \mathrm{CH}$ & $80.1 \mathrm{CH}$ & $79.2 \mathrm{CH}$ & $79.9 \mathrm{CH}$ \\
\hline 8 & $131.6 \mathrm{qC}$ & $132.0 \mathrm{qC}$ & $136.2 \mathrm{qC}$ & $139.0 \mathrm{qC}$ \\
\hline 9 & $132.0 \mathrm{CH}$ & $131.7 \mathrm{CH}$ & $129.4 \mathrm{CH}$ & $129.8 \mathrm{CH}$ \\
\hline 10 & $46.2 \mathrm{CH}$ & $46.1 \mathrm{CH}$ & $45.9 \mathrm{CH}$ & $46.5 \mathrm{CH}$ \\
\hline 11 & $211.5 \mathrm{qC}$ & $211.7 \mathrm{qC}$ & 211.9 qC & $212.7 \mathrm{qC}$ \\
\hline 12 & $42.6 \mathrm{CH}_{2}$ & $42.7 \mathrm{CH}_{2}$ & $42.5 \mathrm{CH}_{2}$ & $44.3 \mathrm{CH}_{2}$ \\
\hline 13 & $68.9 \mathrm{CH}$ & $68.8 \mathrm{CH}$ & $69.0 \mathrm{CH}$ & $69.3 \mathrm{CH}$ \\
\hline 14 & $81.6 \mathrm{qC}$ & $81.6 \mathrm{qC}$ & $81.6 \mathrm{qC}$ & $85.0 \mathrm{qC}$ \\
\hline 15 & $210.8 \mathrm{qC}$ & $211.0 \mathrm{qC}$ & $211.2 \mathrm{qC}$ & $216.5 \mathrm{qC}$ \\
\hline 16 & $46.9 \mathrm{CH}$ & $47.0 \mathrm{CH}$ & $46.9 \mathrm{CH}$ & $46.4 \mathrm{CH}$ \\
\hline 17 & $77.8 \mathrm{CH}$ & $77.7 \mathrm{CH}$ & $77.7 \mathrm{CH}$ & $78.4 \mathrm{CH}$ \\
\hline 18 & $62.7 \mathrm{qC}$ & $62.7 \mathrm{qC}$ & $62.6 \mathrm{qC}$ & $63.9 \mathrm{qC}$ \\
\hline 19 & $67.1 \mathrm{CH}$ & $67.0 \mathrm{CH}$ & $67.0 \mathrm{CH}$ & $67.4 \mathrm{CH}$ \\
\hline 20 & $31.1 \mathrm{CH}$ & $31.1 \mathrm{CH}$ & $30.9 \mathrm{CH}$ & $32.4 \mathrm{CH}$ \\
\hline 21 & $129.7 \mathrm{CH}$ & $129.4 \mathrm{CH}$ & $129.6 \mathrm{CH}$ & $131.6 \mathrm{CH}$ \\
\hline 22 & $125.5 \mathrm{CH}$ & $125.5 \mathrm{CH}$ & $125.4 \mathrm{CH}$ & $126.2 \mathrm{CH}$ \\
\hline 23 & $13.5 \mathrm{CH}_{3}$ & $13.4 \mathrm{CH}_{3}$ & $13.3 \mathrm{CH}_{3}$ & $13.3 \mathrm{CH}_{3}$ \\
\hline 24 & $14.6 \mathrm{CH}_{3}$ & $14.5 \mathrm{CH}_{3}$ & $14.4 \mathrm{CH}_{3}$ & $15.3 \mathrm{CH}_{3}$ \\
\hline 25 & $14.7 \mathrm{CH}_{3}$ & $14.5 \mathrm{CH}_{3}$ & $15.0 \mathrm{CH}_{3}$ & $15.6 \mathrm{CH}_{3}$ \\
\hline 26 & $10.8 \mathrm{CH}_{3}$ & $10.7 \mathrm{CH}_{3}$ & $10.2 \mathrm{CH}_{3}$ & $10.5 \mathrm{CH}_{3}$ \\
\hline 27 & $16.3 \mathrm{CH}_{3}$ & $16.2 \mathrm{CH}_{3}$ & $16.4 \mathrm{CH}_{3}$ & $15.7 \mathrm{CH}_{3}$ \\
\hline 28 & $63.5 \mathrm{CH}_{2}$ & $63.5 \mathrm{CH}_{2}$ & $63.5 \mathrm{CH}_{2}$ & $65.7 \mathrm{CH}_{2}$ \\
\hline 29 & $63.2 \mathrm{CH}_{2}$ & $63.2 \mathrm{CH}_{2}$ & $62.8 \mathrm{CH}_{2}$ & $64.8 \mathrm{CH}_{2}$ \\
\hline 30 & $11.1 \mathrm{CH}_{3}$ & $11.0 \mathrm{CH}_{3}$ & $10.9 \mathrm{CH}_{3}$ & $11.5 \mathrm{CH}_{3}$ \\
\hline 31 & $18.6 \mathrm{CH}_{3}$ & $18.4 \mathrm{CH}_{3}$ & $18.3 \mathrm{CH}_{3}$ & $18.7 \mathrm{CH}_{3}$ \\
\hline 32 & $60.3 \mathrm{CH}_{3}$ & $60.3 \mathrm{CH}_{3}$ & $60.3 \mathrm{CH}_{3}$ & $60.3 \mathrm{CH}_{3}$ \\
\hline 33 & $169.5 \mathrm{qC}$ & $169.9 \mathrm{qC}$ & - & - \\
\hline 34 & $21.6 \mathrm{CH}_{3}$ & $21.5 \mathrm{CH}_{3}$ & - & - \\
\hline 35 & $173.5 \mathrm{qC}$ & $173.7 \mathrm{qC}$ & $173.6 \mathrm{qC}$ & - \\
\hline 36 & $34.2 \mathrm{CH}_{2}$ & $34.1 \mathrm{CH}_{2}$ & $34.0 \mathrm{CH}_{2}$ & - \\
\hline 37 & $29.0 \mathrm{CH}_{2}$ & $29.0 \mathrm{CH}_{2}$ & $29.1 \mathrm{CH}_{2}$ & - \\
\hline
\end{tabular}

${ }^{\text {a }}$ Measured in $\mathrm{CDCl}_{3}$. ${ }^{\mathrm{b}}$ Measured in $\mathrm{CD}_{3} \mathrm{OD}$.

Then, the cells were observed under a microscope to evaluate viability of cells whether the fractions were cytotoxic or not.

2.11. MTT Assay. Cultured cells were inoculated to a 96-well plate with approximate cell density of $1 \times 10^{4}$ cells $/ \mathrm{mL}$ in DMEM. After $24 \mathrm{~h}$ incubation, a series of DMSO solution of compounds 1-3 were applied to each well and the final concentrations were adjusted as $0,1,12.5,25,37.5,50,62.5$, to $75 \mathrm{ng} / \mathrm{mL}$. Cells were incubated for another $24 \mathrm{~h}$, and the media were replaced with $20 \mu \mathrm{L}$ of $5 \mathrm{~g} / \mathrm{mL}$ MTT solution in PBS and incubated for $3.5 \mathrm{~h}$. After removal of PBS solution, an amount of $150 \mu \mathrm{L}$ of DMSO was added to each well and the cells were reincubated for $15 \mathrm{~min}$ prior to measurement with a Tecan microplate reader at $590 \mathrm{~nm}$ with reference filter at $620 \mathrm{~nm}[20,21]$.

\section{Results and Discussion}

As an EtOAc soluble portion of a methanolic extract of the sponge K09-02 showed potent cytotoxicity against cultured NBT-T2 cells, the portion was separated repetitively on a silica gel column followed by Si-60 HPLC affording three compounds 1, 2, and 3 as shown in Figure 1.

By inspecting ${ }^{1} \mathrm{H}$ and ${ }^{13} \mathrm{C}$ NMR spectra of compound 1 together with database search (Tables 1 and 2, Figures S1 and S2 (Supplementary Materials available online 
TABLe 2: ${ }^{1} \mathrm{H}$ NMR data for compounds $\mathbf{1}, \mathbf{2}$, and $\mathbf{3}(\mathrm{J}$ in $\mathrm{Hz}$ ).

\begin{tabular}{|c|c|c|c|c|}
\hline C no. & $\mathbf{1}^{15, \mathrm{a}}$ & $1^{\mathrm{a}}$ & $2^{\mathrm{a}}$ & $3^{\mathrm{b}}$ \\
\hline 1 & - & - & - & - \\
\hline 2 & $3.96 \mathrm{dd}(1.0,7.3)$ & $3.96 \mathrm{dd}(1.3,7.5)$ & $3.98 \mathrm{dd}(1.3,7.8)$ & $3.76 \mathrm{~d}(2.2)$ \\
\hline 3 & $3.64 \mathrm{dd}(1.3,7.8)$ & $3.67 \mathrm{dd}(1.3,8.0)$ & $3.67 \mathrm{dd}(1.3,8.4)$ & $3.81 \mathrm{dd}(2.2,9.8)$ \\
\hline 4 & $3.12 \mathrm{~m}$ & $3.13 \mathrm{dd}(8.0,7.1)$ & $3.10 \mathrm{dq}(8.4,7.3)$ & $3.16 \mathrm{dq}(9.8,7.1)$ \\
\hline 5 & - & - & - & - \\
\hline 6 & $3.18 \mathrm{dq}(10.7,7.3)$ & $3.22 \mathrm{dq}(10.7,6.8)$ & $3.04 \mathrm{dq}(9.8,6.8)$ & $3.16 \mathrm{dq}(10.0,7.1)$ \\
\hline 7 & $5.39 \mathrm{~d}(10.7)$ & $5.41 \mathrm{~d}(10.7)$ & $4.12 \mathrm{~d}(10.0)$ & $4.03 \mathrm{~d}(10.0)$ \\
\hline 8 & - & - & - & - \\
\hline 9 & $5.60 \mathrm{~d}(9.3)$ & $5.62 \mathrm{~d}(9.6)$ & $5.48 \mathrm{~d}(10.5)$ & $5.33 \mathrm{~d}(9.3)$ \\
\hline 10 & $3.38 \mathrm{dq}(9.3,6.8)$ & $3.41 \mathrm{dq}(9.6,7.0)$ & $3.49 \mathrm{dq}(10.5,7.1)$ & $3.36 \mathrm{dq}(9.3,6.8)$ \\
\hline 11 & - & - & - & - \\
\hline \multirow{2}{*}{12} & $2.66 \mathrm{dd}(9.8,16.1)$ & $2.69 \mathrm{dd}(9.8,16.1)$ & $2.72 \mathrm{dd}(9.8,16.1)$ & $2.75 \mathrm{dd}(9.5,17.6)$ \\
\hline & $2.49 \mathrm{dd}(2.4,16.1)$ & $2.49 \mathrm{dd}(2.5,16.1)$ & $2.51 \mathrm{dd}(2.0,16.1)$ & $2.23 \mathrm{dd}(2.0,17.6)$ \\
\hline 13 & $4.40 \mathrm{~m}$ & $4.42 \mathrm{~m}$ & $4.39 \mathrm{dt}(2.0,9.8)$ & $4.44 \mathrm{dd}(2.9,9.5)$ \\
\hline 14 & - & - & - & - \\
\hline 15 & - & - & - & - \\
\hline 16 & $4.02 \mathrm{dt}(3.9,10.9)$ & $\begin{array}{c}4.03 \text { ddd }(3.9,10.5 \\
11.5)\end{array}$ & $4.09 \mathrm{dt}(3.9,11.0)$ & 4.08 ddd $(3.9,10.8,11.4)$ \\
\hline 17 & $3.12 \mathrm{~m}$ & $3.13 \mathrm{~m}$ & $3.20 \mathrm{dd}(11.0)$ & $3.20 \mathrm{~d}(10.7)$ \\
\hline 18 & - & - & - & - \\
\hline 19 & $2.56 \mathrm{~d}(9.3)$ & $2.59 \mathrm{~d}(9.3)$ & $2.58 \mathrm{~d}(9.8)$ & $2.62 \mathrm{~d}(9.3)$ \\
\hline 20 & $2.44 \mathrm{~m}$ & $2.47 \mathrm{~m}$ & $2.47 \mathrm{~m}$ & $2.48 \mathrm{~m}$ \\
\hline 21 & $5.23 \mathrm{dt}(1.5,10.7)$ & $\begin{array}{c}5.25 \text { ddd }(0.7,10.2 \\
10.9)\end{array}$ & $5.24 \mathrm{dt}(1.5,10.5)$ & $5.31 \mathrm{~m}$ \\
\hline 22 & $5.48 \mathrm{dq}(10.7,6.8)$ & $5.51 \mathrm{dq}(10.9,6.8)$ & $5.49 \mathrm{dq}(10.5,6.8)$ & $5.51 \mathrm{dq}(10.7,6.8)$ \\
\hline 23 & $1.59 \mathrm{dd}(1.5,6.8)$ & $1.62 \mathrm{dd}(1.2,6.8)$ & $1.62 \mathrm{dd}(1.5,6.8)$ & $1.62 \mathrm{dd}(1.7,6.8)$ \\
\hline 24 & $1.18 \mathrm{~d}(6.8)$ & $1.21 \mathrm{~d}(7.1)$ & $1.21 \mathrm{~d}(7.3)$ & $1.23 \mathrm{~d}(7.1)$ \\
\hline 25 & $1.13 \mathrm{~d}(7.3)$ & $1.16 \mathrm{~d}(6.8)$ & $1.28 \mathrm{~d}(6.8)$ & $1.26 \mathrm{~d}(7.1)$ \\
\hline 26 & 1.54 brd (1.0) & $1.59 \mathrm{~d}(0.8)$ & $1.63 \mathrm{~s}$ & $1.65 \mathrm{~d}(1.5)$ \\
\hline 27 & $1.07 \mathrm{~d}(6.8)$ & $1.09 \mathrm{~d}(7.0)$ & $1.10 \mathrm{~d}(7.1)$ & $1.03 \mathrm{~d}(6.8)$ \\
\hline \multirow{2}{*}{28} & $4.44 \mathrm{~d}(11.7)$ & $4.46 \mathrm{~d}(11.5)$ & $4.45 \mathrm{~d}(11.5)$ & $3.75 \mathrm{~d}(10.5)$ \\
\hline & $4.19 \mathrm{~d}(11.7)$ & $4.22 \mathrm{~d}(11.5)$ & $4.21 \mathrm{~d}(11.5)$ & $3.76 \mathrm{~d}(10.5)$ \\
\hline \multirow{2}{*}{29} & $4.17 \mathrm{dd}(3.7,9.8)$ & $4.20 \mathrm{dd}(3.7,10.2)$ & $4.24 \mathrm{dd}(3.0,9.5)$ & $4.35 \mathrm{dd}(3.9,10.5)$ \\
\hline & $4.10 \mathrm{dd}(10.2,10.9)$ & $4.12 \mathrm{dd}(10.2,11.2)$ & $4.08 \mathrm{~m}$ & $3.91 \mathrm{dd}(10.5,11.4)$ \\
\hline 30 & $1.38 \mathrm{~s}$ & $1.42 \mathrm{~s}$ & $1.42 \mathrm{~s}$ & $1.35 \mathrm{~s}$ \\
\hline 31 & $1.11 \mathrm{~d}(6.4)$ & $1.13 \mathrm{~d}(6.3)$ & $1.14 \mathrm{~d}(6.6)$ & $1.11 \mathrm{~d}(6.6)$ \\
\hline 32 & $3.28 \mathrm{~s}$ & $3.31 \mathrm{~s}$ & $3.30 \mathrm{~s}$ & $3.39 \mathrm{~s}$ \\
\hline 33 & - & - & - & - \\
\hline 34 & $2.01 \mathrm{~s}$ & $2.04 \mathrm{~s}$ & - & - \\
\hline 35 & - & - & - & - \\
\hline 36 & $2.24 \mathrm{t}(7.6)$ & $2.27 \mathrm{t}(7.6)$ & $2.27 \mathrm{t}(11.8)$ & \\
\hline 37 & $1.53 \mathrm{brs}$ & $1.59 \mathrm{brs}$ & 1.25 brs & \\
\hline $\mathrm{OH}-2$ & $2.85 \mathrm{~d}(7.3)$ & $2.92 \mathrm{~d}(7.5)$ & $2.99 \mathrm{~d}(7.8)$ & $2.92 \mathrm{~d}(7.5)$ \\
\hline $\mathrm{OH}-13$ & - & & $4.71 \mathrm{~s}$ & \\
\hline
\end{tabular}

${ }^{\mathrm{a}}$ Measured in $\mathrm{CDCl}_{3}$. ${ }^{\mathrm{b}}$ Measured in $\mathrm{CD}_{3} \mathrm{OD}$. 


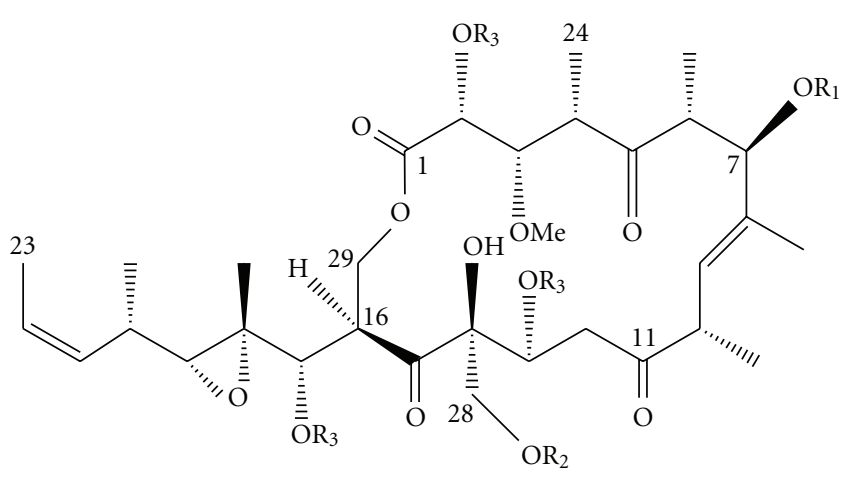

(1) $\mathrm{R}_{1}=\mathrm{Ac}, \mathrm{R}_{2}=\mathrm{CO}\left(\mathrm{CH}_{2}\right)_{14-16} \mathrm{CH}_{3}, \mathrm{R}_{3}=\mathrm{H}$

(2) $\mathrm{R}_{1}=\mathrm{R}_{3}=\mathrm{H}, \mathrm{R}_{2}=\mathrm{CO}\left(\mathrm{CH}_{2}\right)_{14-19} \mathrm{CH}_{3}$

(3) $\mathrm{R}_{1}=\mathrm{R}_{2}=\mathrm{R}_{3}=\mathrm{H}$

(4) $\mathrm{R}_{1}=\mathrm{R}_{3}=\mathrm{Ac}, \mathrm{R}_{2}=\mathrm{CO}\left(\mathrm{CH}_{2}\right)_{14-16} \mathrm{CH}_{3}$

FIGURE 1: Structures of compounds (1)-(4).

at doi:10.5402/2011/852619)), we could readily identify that it is a member of candidaspongiolide, a series of 18membered cytotoxic macrolide retaining one of fatty acid moieties from $\mathrm{C}_{14}$ to $\mathrm{C}_{18}$ at C-28 [15]. HR-ESIMS of our material exhibited molecular-related ions at $\mathrm{m} / \mathrm{z}$ 945.55514, 959.57079, $973.58884[\mathrm{M}+\mathrm{Na}]^{+}$indicating that compound $\mathbf{1}$ is candidaspongiolide esterified with the homologs of three saturated fatty acids (palmitic, margaric, and stearic acids).

Compound 2 was obtained as a colorless glass with $[\alpha]_{D}^{25}$ +72. After elucidation of its ${ }^{1} \mathrm{H}$ and ${ }^{13} \mathrm{C}$ NMR spectra, compound 2 was found to be an analog of 1 . However, the ${ }^{13} \mathrm{C}$ NMR spectrum showed two carbonyl carbons at $\delta_{\mathrm{C}}$ $171.3 \mathrm{q}(\mathrm{C}-1)$ and 173.1 (C-35) instead of three in $\mathbf{1}$ (Table 1, Figure S3). As the signals for an acetoxy group $\left(\delta_{\mathrm{H}} 2.04 \mathrm{~s}\right.$, $\left.\delta_{\mathrm{C}} 21.5 \mathrm{q}\right)$ in $\mathbf{1}$ are missing in $\mathbf{2}$, it was suggested that $\mathbf{2}$ is a deacetyl derivative of $\mathbf{1}$. The lack of the acetyl group is in a good agreement with ${ }^{1} \mathrm{H}$ NMR spectrum and COSY analysis showing that $\mathrm{H}-7$ proton signal $\left(\delta_{\mathrm{H}} 4.12 \mathrm{~d}, J=\right.$ $10.0 \mathrm{~Hz})$ in 2 shifted to higher field than that $\left(\delta_{\mathrm{H}} 5.41 \mathrm{~d}, J\right.$ $=10.7 \mathrm{~Hz}$ ) in $\mathbf{1}$ (Table 2). HR-ESIMS of 2 showed a series of sodiated ions $[\mathrm{M}+\mathrm{Na}]^{+}$at $\mathrm{m} / \mathrm{z} 903.54964,917.56023$, $931.57576,945.60036$, and 959.61056 corresponding to the presence of $\mathrm{C}_{16}$ to $\mathrm{C}_{20}$ esters. For structural confirmation, compound 2 was acetylated to give tetraacetate 4 , which showed signals identical with 4 obtained from 1 (Figure S4). Compound 4 exhibited four acetyl signals at $\delta_{\mathrm{H}} 2.22 \mathrm{~s}, 2.10 \mathrm{~s}$, $2.09 \mathrm{~s}$, and $2.02 \mathrm{~s}$ and molecular-related ions corresponding to macrolide esters with $\mathrm{C}_{16}$ to $\mathrm{C}_{18}$ fatty acids.

Compound 3 was isolated as a yellowish glass with $[\alpha]_{D}^{25}$ +97. Its molecular formula was established as $\mathrm{C}_{32} \mathrm{H}_{50} \mathrm{O}_{13}$ by observing a molecular-related ion at $\mathrm{m} / \mathrm{z} 665.31522$ $[\mathrm{M}+\mathrm{Na}]^{+}$in HR-ESIMS. ${ }^{1} \mathrm{H}$ and ${ }^{13} \mathrm{C}$ NMR spectra (Tables 1 and 2, Figures S5 and S6) revealed that compound 3 has a similar macrolide structure to that of compound 1 except for the lack of a fatty acid ester moiety and an acetate found in 1. Higher field chemical shifts observed for H-7 $\left(\delta_{\mathrm{H}} 4.03\right)$ and $\mathrm{H}-28\left(\delta_{\mathrm{H}} 3.75\right)$ indicated that 3 is devoid of acyl groups. Close similarity of ${ }^{1} \mathrm{H}$ and ${ }^{13} \mathrm{C}$ NMR data of $\mathbf{3}$ to $\mathbf{1}$ (Table 2) indicated that the macrolide core structure of compound $\mathbf{3}$ is identical to compound $\mathbf{1}$.

All of natural compounds 1-3 exhibited potent cytotoxicity, $\mathrm{IC}_{50} 37,4.7$, and $19 \mathrm{ng} / \mathrm{mL}$, against NBT-T2 cells. The result is not in good agreement with those reported by Meragelman and coworkers, that is, candidaspongiolide (1) showed stronger growth inhibition $\left(\mathrm{GI}_{50} 14 \mathrm{ng} / \mathrm{mL}\right)$ than the core compound $(42 \mathrm{ng} / \mathrm{mL})$ [15]. Additionally Paul et al. paperd the importance of a linear carbon chain on the cytotoxicity in the case of amphidinol [22]. The difference may be explained either by the number of cell lines or by different sensitivity of NBT-T2 cells.

\section{Acknowledgments}

The authors appreciate Professor Rob van Soest, University of Amsterdam, for showing a sponge image and suggestions on the sponge identification and $\mathrm{Mr}$. T. Iha for mass measurements. they thank PharmaMar S. A. for financial support.

\section{References}

[1] R. Ruzicka and D. F. Gleason, "Sponge community structure and anti-predator defenses on temperate reefs of the South Atlantic Bight," Journal of Experimental Marine Biology and Ecology, vol. 380, no. 1-2, pp. 36-46, 2009.

[2] M. Pfannkuchen, G. B. Fritz, S. Schlesinger, K. Bayer, and F. Brümmer, "In situ pumping activity of the sponge Aplysina aerophoba, Nardo 1886," Journal of Experimental Marine Biology and Ecology, vol. 369, no. 1, pp. 65-71, 2009.

[3] M. Assmann, R. W. M. van Soest, and M. Köck, "New antifeedant bromopyrrole alkaloid from the caribbean sponge Stylissa caribica," Journal of Natural Products, vol. 64, no. 10, pp. 1345-1347, 2001.

[4] A. Vik, E. Hedner, C. Charnock et al., “(+)-Agelasine D: improved synthesis and evaluation of antibacterial and cytotoxic activities," Journal of Natural Products, vol. 69, no. 3, pp. 381-386, 2006.

[5] C. Boonlarppradab and D. J. Faulkner, "Eurysterols A and B, cytotoxic and antifungal steroidal sulfates from a marine sponge of the genus Euryspongia," Journal of Natural Products, vol. 70, no. 5, pp. 846-848, 2007.

[6] M. Sjögren, U. Göransson, A. L. Johnson et al., "Antifouling activity of brominated cyclopeptides from the marine sponge Geodia barretti," Journal of Natural Products, vol. 67, no. 3, pp. 368-372, 2004.

[7] S. Nishimura, S. Matsunaga, S. Yoshida, Y. Nakao, H. Hirota, and N. Fusetani, "Structure-activity relationship study on 13-deoxytedanolide, a highly antitumor macrolide from the marine sponge Mycale adhaerens," Bioorganic and Medicinal Chemistry, vol. 13, no. 2, pp. 455-462, 2005.

[8] A. Longeon, B. R. Copp, M. Roué et al., "New bioactive halenaquinone derivatives from South Pacific marine sponges of the genus Xestospongia," Bioorganic and Medicinal Chemistry, vol. 18, no. 16, pp. 6006-6011, 2010.

[9] E. H. Belarbi, A. C. Gómez, Y. Chisti, F. G. Camacho, and E. M. Grima, "Producing drugs from marine sponges," Biotechnology Advances, vol. 21, no. 7, pp. 585-598, 2003. 
[10] F. J. Schmitz, S. P. Gunasekera, G. Yalamanchili, M. B. Hossain, and D. van der Helm, "Tedanolide: a potent cytotoxic macrolide from the Caribbean sponge Tedania ignis," Journal of the American Chemical Society, vol. 106, no. 23, pp. 72517252, 1984.

[11] C. Chevallier, T. S. Bugni, X. Feng, M. K. Harper, A. M. Orendt, and C. M. Ireland, "Tedanolide C: a potent new 18membered-ring cytotoxic macrolide isolated from the Papua New Guinea marine sponge Ircinia sp," Journal of Organic Chemistry, vol. 71, no. 6, pp. 2510-2513, 2006.

[12] A. B. Smith and S. A. Lodise, "Synthesis of tedanolide and 13-deoxytedanolide. Assembly of a common C(1)-C(11) subtarget," Organic Letters, vol. 1, no. 8, pp. 1249-1252, 1999.

[13] M. E. Jung and R. Marquez, "Efficient synthesis of the C1C11 fragment of the tedanolides. The nonaldol aldol process in synthesis," Organic Letters, vol. 2, no. 12, pp. 1669-1672, 2000.

[14] G. Ehrlich, J. Hassfeld, U. Eggert, and M. Kalesse, "The total synthesis of (+)-tedanolide," Journal of the American Chemical Society, vol. 128, no. 43, pp. 14038-14039, 2006.

[15] T. L. Meragelman, R. H. Willis, G. M. Woldemichael et al., "Candidaspongiolides, distinctive analogues of tedanolide from sponges of the genus Candidaspongia," Journal of Natural Products, vol. 70, no. 7, pp. 1133-1138, 2007.

[16] D. Trisciuoglio, B. Uranchimeg, J. H. Cardellina et al., "Induction of apoptosis in human cancer cells by candidaspongiolide, a novel sponge polyketide," Journal of the National Cancer Institute, vol. 100, no. 17, pp. 1233-1246, 2008.

[17] N. Hanif, J. Tanaka, A. Setiawan et al., "Polybrominated diphenyl ethers from the Indonesian sponge Lamellodysidea herbacea," Journal of Natural Products, vol. 70, no. 3, pp. 432435, 2007.

[18] S. Aratake, A. Trianto, N. Hanif, N. J. de Voogd, and J. Tanaka, "A new polyunsaturated brominated fatty acid from a Haliclona sponge," Marine Drugs, vol. 7, no. 4, pp. 523-527, 2009.

[19] C. J. Herman, P. D. Vegt, F. M. Debruyne, G. P. Vooijs, and F. C. Ramaekers, "Squamous and transitional elements in rat bladder carcinomas induced by N-butyl-N-4-hydroxybutylnitrosamine (BBN): a study of cytokeratin expression," American Journal of Pathology, vol. 120, no. 3, pp. 419-426, 1985.

[20] E. Ulukaya, F. Ozdikicioglu, A. Y. Oral, and M. Demirci, “The MTT assay yields a relatively lower result of growth inhibition than the ATP assay depending on the chemotherapeutic drugs tested," Toxicology in Vitro, vol. 22, no. 1, pp. 232-239, 2008.

[21] http://www.brc.riken.jp/lab/cell .

[22] G. K. Paul, N. Matsumori, K. Konoki, M. Murata, and K. Tachibana, "Chemical structures of amphidinols 5 and 6 isolated from marine dinoflagellate Amphidinium klebsii and their cholesterol-dependent membrane disruption," Journal of Marine Biotechnology, vol. 5, no. 2-3, pp. 124-128, 1997. 

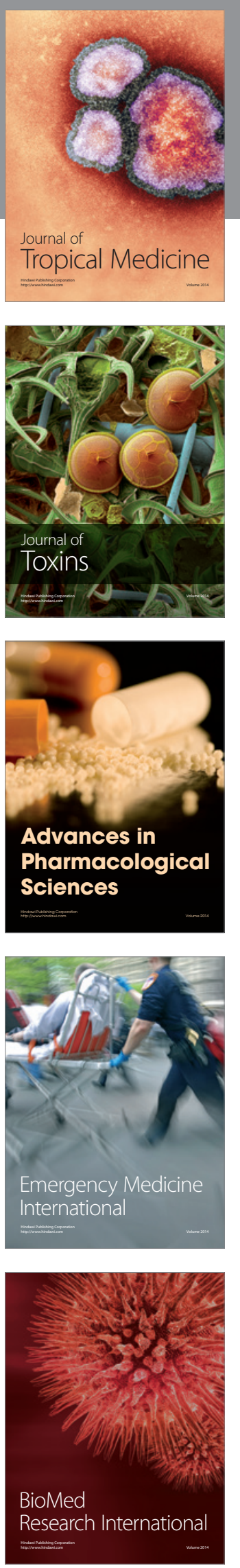
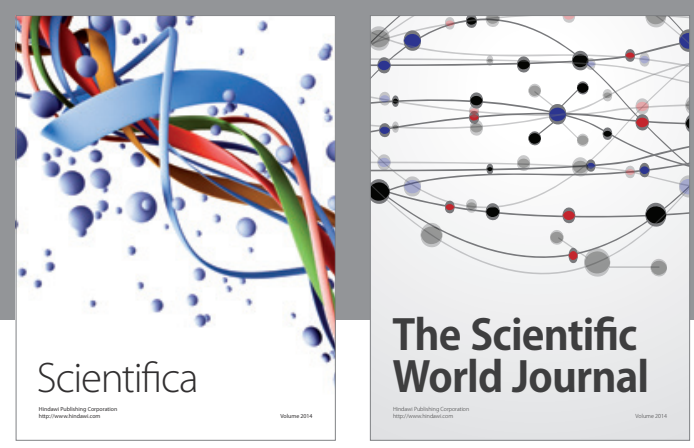

The Scientific World Journal
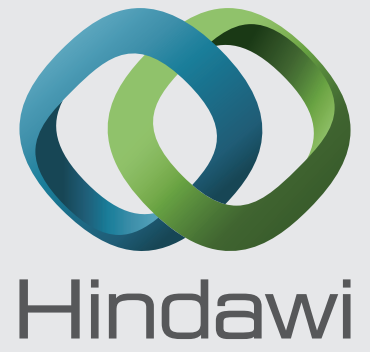

Submit your manuscripts at

http://www.hindawi.com
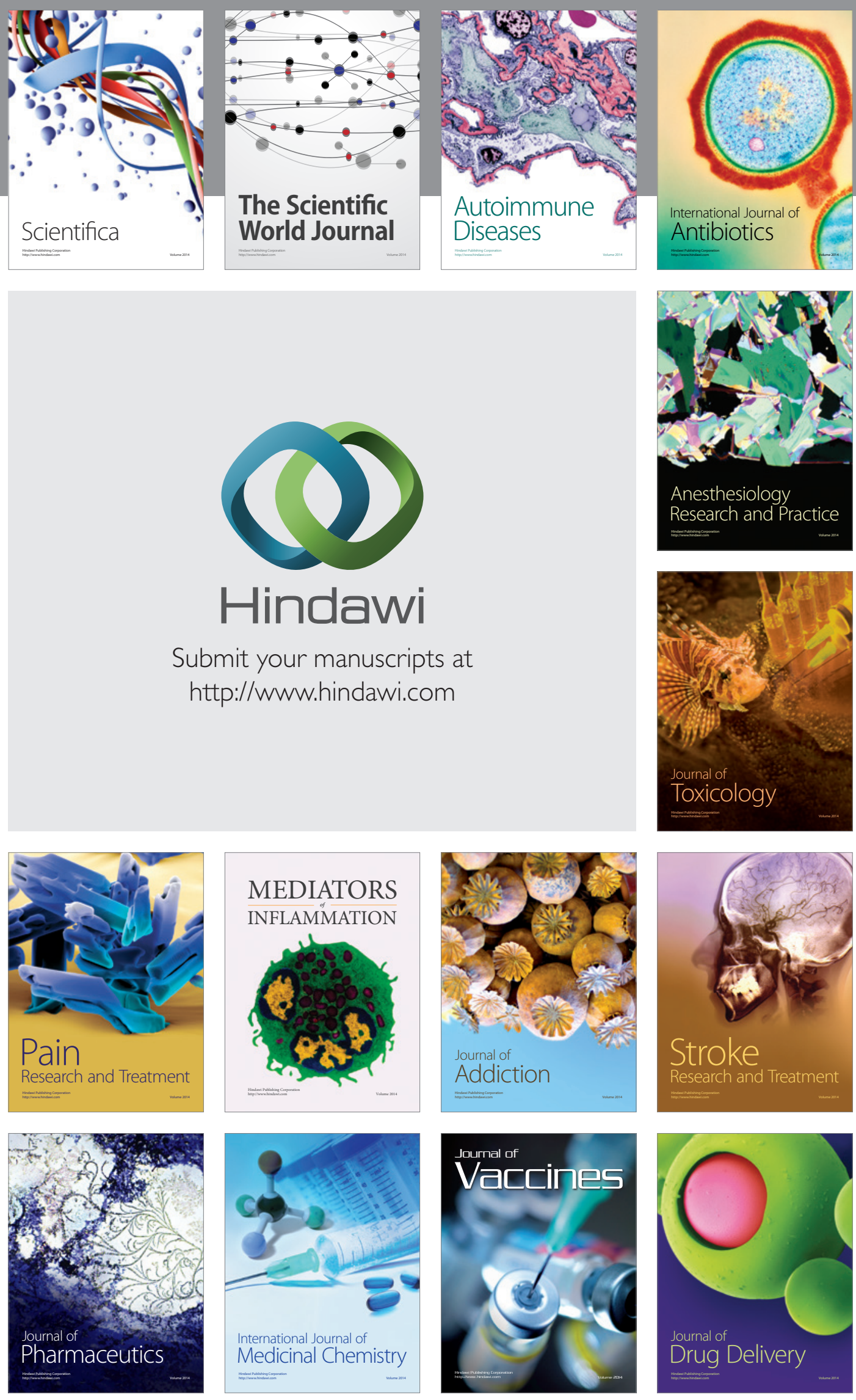